\title{
Topological Anderson insulator phenomena
}

\author{
Yanxia Xing, ${ }^{1,2}$ Lei Zhang, ${ }^{2}$ and Jian Wang, ${ }^{2, *}$ \\ ${ }^{1}$ Department of Physics, Beijing Institute of Technology, Beijing 100081, China \\ ${ }^{2}$ Department of Physics and the Center of Theoretical and Computational Physics, The University of Hong Kong, \\ Pokfulam Road, Hong Kong, China
}

(Received 5 March 2011; revised manuscript received 29 April 2011; published 20 July 2011)

\begin{abstract}
We study the nature of the disorder-induced quantized conductance, i.e., the phenomena of topological Anderson insulator (TAI). The disorder effect in several different systems where the anomalous Hall effect exists is numerically studied using the tight-binding Hamiltonian. It is found that the TAI phenomena can also exist in the modified Dirac model where the quadratic corrections $k^{2} \sigma_{z}$ are included and the electron-hole symmetry is kept. These phenomena also occur in the graphene system with the next-nearest-neighbor coupling and the staggered sublattice potential. For the graphene sheet with Rashba spin-orbit interaction as well as an exchange field, a precursor of TAI is observed. A comparison between the localization length of the two-dimensional ribbon and two-dimensional cylinder structures clearly reveals the topological nature of these phenomena. Furthermore, analysis on the local current density in anomalous quantum Hall systems where the TAI phenomena may or may not arise reveals the nature of TAI phenomena. In the presence of small disorders, the conductance is not quantized and the bulk and edge states coexist in the system. As disorder strength increases, the bulk state is quickly destroyed, while the robust edge state may survive. When the edge state is robust enough to sustain the strong disorder that completely kills the bulk state, TAI phenomena arise.
\end{abstract}

DOI: 10.1103/PhysRevB.84.035110

PACS number(s): 73.23.-b, 73.20.At, 72.15.Rn

\section{INTRODUCTION}

It is known that the two-dimensional (2D) noninteracting system with the quadratic dispersion relation is an Anderson insulator. ${ }^{1}$ In the presence of strong spin-orbit coupling (SOC), ${ }^{2,3}$ the external ${ }^{3,4}$ or internal ${ }^{5}$ magnetic field, the metallic state can exist at small enough disorders. Due to the crossing of the mobility edge, ${ }^{6}$ a metal-insulator transition (MIT) occurs at the critical disorder strength $w_{c}$ where the localization length is divergent. Recently, $\mathrm{Li}$ et $a{ }^{7}{ }^{7}$ found that the disorder drives either a metallic state or an ordinary insulating state to the topological insulator ${ }^{8}$ (so-called topological Anderson insulator) in the $\mathrm{HgTe} / \mathrm{CdTe}$ quantum well, which has been numerically confirmed by Jiang et al. ${ }^{9}$ By using the effective medium theory, the mechanism of the TAI is explained as the crossing of a band edge rather than a mobility edge by Groth et al. ${ }^{10}$ Although there are many investigations focused on the TAI, there are still some unanswered questions. For instance, what leads to the bandedge crossing? What's the nature of the TAI phenomena (i.e., disorder-induced quantized conductance)? Furthermore, except for the HgTe/CdTe quantum well, are there any other systems that exhibit TAI phenomena? What's the necessary condition to generate TAI phenomena? It is the purpose of this paper to address these questions.

In a $2 \mathrm{D}$ anomalous quantum Hall ${ }^{11,12}$ system, the topological edge states connect the energetically separated continuum of energy band, and only the unidirectional topological edge state contributes to the conductance in the band gap. Due to the topological stability of Chern numbers, ${ }^{13}$ the conductance remains quantized in the presence of weak disorder. At strong disorders, the mobility edges are crossed, and MIT ${ }^{4,6}$ occurs. However, outside of the gap, the bulk state and edge state may coexist. Because the edge state is robust against disorders, it could happen that the bulk state is completely killed before the edge state is destroyed, which may lead to the quantized conductance plateau. The quantized value is determined by the number of the robust edge states. Taking into account the edge state tied to both the spin up and spin down, disorder-induced TAI can be formed, which is confirmed by our calculations on the modified Dirac model. So, roughly speaking, as long as the bulk states and the edge state coexist in the system, disorder-induced quantized conductance can emerge if the edge state sustains when bulk states are destroyed by disorders.

To study the nature of TAI phenomena found in Ref. 7 for the $\mathrm{HgTe} / \mathrm{CdTe}$ quantum well, ${ }^{14}$ we find two different models in which TAI phenomena also exist. The first model is a modified Dirac model on a square lattice with the quadratic corrections $k^{2} \sigma_{z}$ included and electron-hole symmetry kept. This model is similar to the $\mathrm{HgTe} / \mathrm{CdTe}$ quantum well model except the $e$ - $h$ symmetry is broken in the $\mathrm{HgTe} / \mathrm{CdTe}$ quantum well. ${ }^{14}$ The second model is a graphene model on a honeycomb lattice with the next-nearest-neighbor coupling and a staggered sublattice potential. ${ }^{5}$ In this model, the $e-h$ symmetry and inversion symmetry are all broken, and the antidirectional topological edge states tied to two opposite edges are asymmetrically distributed. Our calculation shows that, in the first and second models, moderate disorder induces a transition from an ordinary metallic state to a TAI with the quantized conductance of $G_{0}=e^{2} / h$. To examine the nature of edge states, we have used two different kinds of boundary conditions: hard wall and periodic boundary conditions, which also correspond to ribbon and cylinder structures. In the disorder-induced quantized conductance regime, the localization length of the ribbon structure is much longer compared to that of the cylinder structure, which clearly reveals the topological origin of the transition. When the disorder is very strong, the mobility edge crosses and MIT occurs. In order to vividly show how the transport electron is scattered by the disorder, we calculate the individual local current density from all channels including the edge-state and bulk-state channels. In addition to the first and second models, 
we also calculate local current density in a third model: the honeycomb graphene system, ${ }^{11}$ in which we consider Rashba spin-orbit interaction, exchange energy, and staggered sublattice potential. In the third model, the conductance is not quantized in the presence of disorders, but weakly depends on the disorder strength. However, the analysis of the eigentransmission channel of edge and bulk states shows the signature of TAI. It is possible that a suitable choice of system parameters can give rise to TAI. For all three models, we find that the disorder kills all the bulk current and induces edge current with unidirection. This means that, in the process of transport, due to the topological nature, the edge state survives at all times. So, in the system where the edge and bulk states coexist, in the moderate disorder, the edge state is partially killed while the bulk states are gradually killed, leading to the flattened conductance. If the edge state is robust enough to resist the disorder that is so strong that the bulk states are completely killed, quantized conductance can be formed.

The rest of the paper is organized as follows. In Sec. II, the Hamiltonian of three-model systems in the tight-binding representation is introduced. The formalisms for calculating the conductance and the local current density vector are then derived. Section III gives numerical results along with some discussions. Finally, a brief summary is presented in Sec. IV.

\section{MODELS AND FORMALISM}

\section{A. Three-model Hamiltonian}

The first model is the modified Dirac model with quadratic corrections $k^{2} \sigma_{z}$, which has the form

$$
\begin{gathered}
H_{1}=\sum_{\mathbf{k}}\left[H_{\uparrow}(\mathbf{k})+H_{\downarrow}(\mathbf{k})\right], H_{\downarrow}(\mathbf{k})=H_{\uparrow}^{*}(-\mathbf{k}), \\
H_{\uparrow}(\mathbf{k})=A\left(\mathbf{k}_{x} \sigma_{x}-\mathbf{k}_{y} \sigma_{y}\right)+(m+B \mathbf{k} \cdot \mathbf{k}) \sigma_{z}+\epsilon(r) \sigma_{0}
\end{gathered}
$$

where $\sigma_{x, y, z}$ are Pauli matrices representing the pseudospin formed by $s, p$ orbitals. In Eq. (1), the momentum $k$ is a good quantum number for periodic systems. This model is similar to the low-energy effective Hamiltonian of a $\mathrm{HgTe} / \mathrm{CdTe}$ quantum well ${ }^{14}$ except that the $e-h$ symmetry is kept here, while it is broken in the $\mathrm{HgTe} / \mathrm{CdTe}$ quantum well. The tight-binding Hamiltonian in square lattice is given by ${ }^{7,9}$

$$
\begin{aligned}
H_{\uparrow}= & \sum_{\mathbf{i}} d_{\mathbf{i}}^{\dagger}\left(\epsilon_{\mathbf{i}} \sigma_{0}+C \sigma_{z}\right) d_{\mathbf{i}} \\
& +\sum_{\mathbf{i}} d_{\mathbf{i}}^{\dagger}\left(t \sigma_{z}-i \frac{A}{2 a} \sigma_{x}\right) d_{\mathbf{i}+\mathbf{a}_{x}}+\text { H.c. } \\
& +\sum_{\mathbf{i}} d_{\mathbf{i}}^{\dagger}\left(t \sigma_{z}+i \frac{A}{2 a} \sigma_{y}\right) d_{\mathbf{i}+\mathbf{a}_{y}}+\text { H.c. }
\end{aligned}
$$

where $\sigma_{0}$ is a $2 \times 2$ unity matrix, $\epsilon_{\mathbf{i}}$ is a random on-site potential (which is uniformly distributed in the region $[-w / 2, w / 2]$ ), $\mathbf{i}=\left(\mathbf{i}_{x}, \mathbf{i}_{y}\right)$ is the index of the discrete site of the system sketched in Fig. 1(a) on the square lattice, and $\mathbf{a}_{x}=[a, 0]$ and $\mathbf{a}_{y}=[0, a]$ are the unit vectors of the square lattice with the lattice constant $a \cdot d_{\mathbf{i}}=\left[d_{s, \mathbf{i}}, d_{p, \mathbf{i}}\right]^{T}$, with $T$ denoting transpose, and $d_{s(p), \mathbf{i}}$ and $d_{s(p), \mathbf{i}}^{\dagger}$ are the annihilation and creation operators for the $s(p)$ orbital at site i. Here, $C=m-4 t$ and $t=B / a^{2}$ denote the nearest-neighbor coupling strength. $\epsilon_{\mathbf{i}}$ in the first term of Eq. (2) is the on-site random energy accounting for the
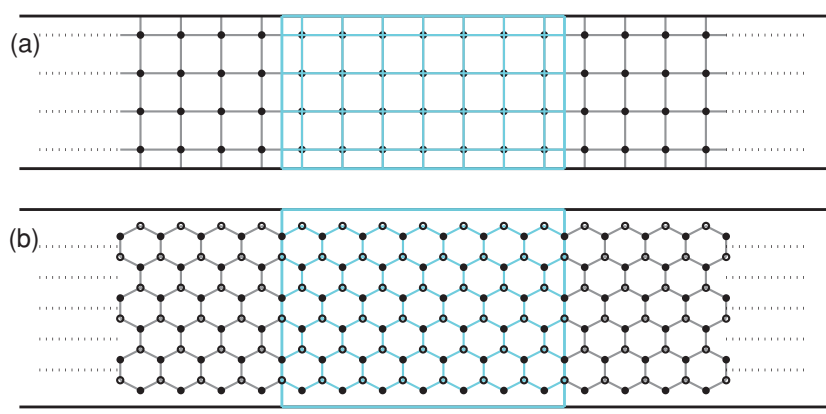

FIG. 1. (Color online) A schematic diagram of the infinite long ribbon on the square lattice for the first model (a) and the infinite long ribbon in the honeycomb lattice along the zigzag direction for the second and third models (b).

disorder. The second term in Eq. (2) is the linear Dirac term, in which the $\sigma_{z}$ involved terms are the quadratic corrections to the Dirac Hamiltonian. The individual spin-up Hamiltonian $H_{\uparrow}$ and spin-down Hamiltonian $H_{\downarrow}$ in Eq. (2) are time-reversal symmetric to each other. Since they are decoupled, we can deal with them individually. So, we shall focus only on the spin-up Hamiltonian $H_{\uparrow}$ in the following calculation.

The second model is proposed by Haldane, which considers the graphene with next-nearest-neighbor coupling and staggered sublattice potential, the Hamiltonian of which can be expressed as ${ }^{5}$

$$
\begin{array}{r}
H_{2}(\mathbf{k})=-t \sum_{i}\left[\epsilon(\mathbf{r}) \sigma_{0}+t_{o} \sigma_{z}+\cos \left(\mathbf{k} \cdot \mathbf{a}_{i}\right) \sigma_{x}-\sin \left(\mathbf{k} \cdot \mathbf{a}_{i}\right) \sigma_{y}\right] \\
+2\left|t_{n}\right|\left[\cos \phi \sum_{i} \cos \left(\mathbf{k} \cdot \mathbf{b}_{i}\right) \sigma_{0}-\sin \phi \sum_{i} \sin \left(\mathbf{k} \cdot \mathbf{b}_{i}\right) \sigma_{z}\right],
\end{array}
$$

where $\sigma_{x, y, z}$ are the Pauli matrices denoting the pseudospin formed by $A B$ sublattice, three nearest-neighbor unit vectors $\mathbf{a}_{i=1,2,3}$ and next-nearest-neighbor unit vectors $\mathbf{b}_{i=1,2,3}$ are given by $\mathbf{a}_{1}=a(0,1), \quad \mathbf{a}_{2}=a(-\sqrt{3} / 2,-1 / 2), \quad \mathbf{a}_{3}=$ $a(\sqrt{3} / 2,-1 / 2), \quad \mathbf{b}_{1}=b(1,0), \quad \mathbf{b}_{2}=b(-1 / 2, \sqrt{3} / 2), \quad \mathbf{b}_{3}=$ $b(-1 / 2,-\sqrt{3} / 2)$ with $a=0.142 \mathrm{~nm}$ and $b=\sqrt{3} a$ denoting the distance between nearest-neighbor sites (lattice constant) and next-nearest-neighbor sites, respectively. In Eq. (3), $t_{o}$ is the staggered sublattice potential, $t=2 \hbar v_{F} / 3 a$ is the nearestneighbor coupling strength with Fermi velocity $v_{F}=0.89 \times$ $10^{6} \mathrm{~ms}^{-1}$, and the next-nearest neighbor coupling $t_{n}=\left|t_{n}\right| e^{i \phi}$ where $\left|t_{n}\right|$ and $\phi$ are the coupling strength and phase deduced from the effective internal magnetic field $B(\mathbf{r})$ along the $\vec{z}$ direction. Here, $t_{n}$ destroys the $e-h$ symmetry of the energy bands as shown in Fig. 2(b) and $B(\mathbf{r})$ breaks the time-reversal invariance. Equation (3) is similar to Eq. (1), which includes all the $\sigma_{x, y, z}$ terms that are $k$ dependent. However, they have different symmetries, e.g., in Eq. (3), both time-reversal and $e-h$ symmetries are broken. In the tight-binding representation, Eq. (3) can be expressed as

$$
\begin{aligned}
H_{2}= & \sum_{\mathbf{i}} d_{\mathbf{i}}^{\dagger}\left(t_{o} \sigma_{z}+\varepsilon_{\mathbf{i}}\right) d_{\mathbf{i}}+t_{c} \sum_{\mathbf{i}, i}\left(a_{\mathbf{i}}^{\dagger} b_{\mathbf{i}+\mathbf{a}_{i}}+\text { H.c. }\right) \\
& +\left|t_{n}\right| \sum_{\mathbf{i}, i}\left[e^{i \phi}\left(a_{\mathbf{i}}^{\dagger} a_{\mathbf{i}+\mathbf{b}_{i}}-b_{\mathbf{i}}^{\dagger} b_{\mathbf{i}+\mathbf{b}_{i}}\right)\right]+\text { H.c. }
\end{aligned}
$$




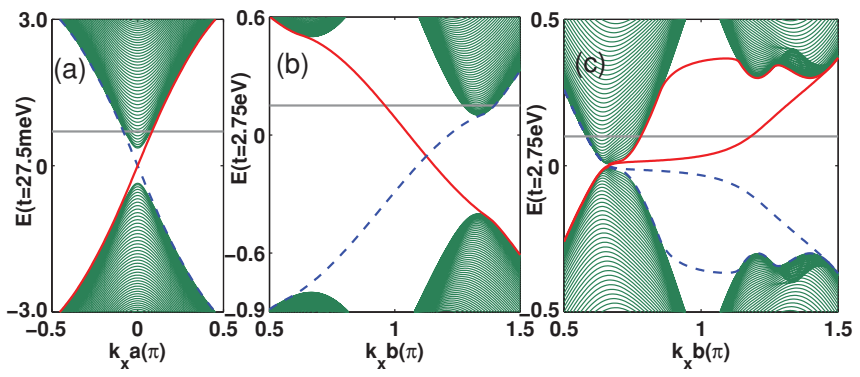

FIG. 2. (Color online) The band structure for the (a) first, (b) second, and (c) third models. The horizontal gray lines marked the Fermi energy $E_{F}=0.7,0.15$, and 0.1 in the three models, respectively. The blue (dark gray in print) dashed lines denote the edge state located in the down edge, and the red (light gray) solid lines are for the edge state located in the up edge.

where $\mathbf{i}=\left(\mathbf{i}_{x}, \mathbf{i}_{y}\right)$ is the index of the discrete site of the honeycomb lattice, which includes two sublattice $A$ (open circle) and $B$ (filled circle) as sketched in Fig. 1(b). $\varepsilon_{\mathbf{i}}=$ $\operatorname{diag}\left(\epsilon_{\mathbf{i}}, \epsilon_{\mathbf{i}+\mathbf{a}_{1}}\right)$ denotes the random potential induced by disor$\operatorname{der} d_{\mathbf{i}}=\left[a_{\mathbf{i}}, b_{\mathbf{i}+\mathbf{a}_{1}}\right]^{T}$, and $a_{\mathbf{i}}\left(b_{\mathbf{i}}\right)$ and $a_{\mathbf{i}}^{\dagger}\left(b_{\mathbf{i}}^{\dagger}\right)$ are the annihilation and creation operators for sublattice $A(B)$ at site $\mathbf{i}$.

In the third model, we consider a graphene sheet with Rashba spin-orbit interaction $\lambda \vec{e}_{z} \cdot(\mathbf{k} \times \mathbf{s})$ as well as an exchange field. ${ }^{11}$ In the tight-binding representation on the honeycomb lattice, corresponding to three directions of translational symmetry, we have $\mathbf{k}_{\mathbf{i}=\mathbf{1 , 2 , 3}} \rightarrow-i \partial_{\mathbf{i}}{ }^{15}$ Projecting them to the $x$ and $y$ directions, we can get $\mathbf{k}_{x}$ and $\mathbf{k}_{y}$. Then, the Hamiltonian of the third graphene model including Rashba spin-orbit coupling, the staggered sublattice potential, and the exchange term can be expressed in the tight-binding representation in the form ${ }^{16}$

$$
\begin{aligned}
H_{3}= & \sum_{\mathbf{i}} d_{\mathbf{i}}^{\dagger}\left(t_{o} \sigma_{z} \otimes s_{0}+t_{e} \sigma_{0} \otimes s_{z}+\varepsilon_{\mathbf{i}}\right) d_{\mathbf{i}} \\
& +\sum_{\mathbf{i}, i} a_{\mathbf{i}}^{\dagger}\left[t_{c} s_{0}+i t_{r} \vec{e}_{z} \cdot\left(\mathbf{s} \times \overrightarrow{\mathbf{a}}_{i}\right)\right] b_{\mathbf{i}+\mathbf{a}_{i}}+\text { H.c. },
\end{aligned}
$$

where $s_{0}$ is the unitary matrix in spin space $\mathbf{s}=\left(s_{x}, s_{y}\right), s_{x, y, z}$ are Pauli matrices denoting the real spin, $d_{\mathbf{i}}^{\dagger}=\left[a_{\mathbf{i}}^{\dagger}, b_{\mathbf{i}+\mathbf{a}_{1}}^{\dagger}\right]$, $a_{\mathbf{i}}^{\dagger}=\left[a_{\uparrow, \mathbf{i}}^{\dagger}, a_{\downarrow, \mathbf{i}}^{\dagger}\right]$, and $b_{\mathbf{i}}^{\dagger}=\left[b_{\uparrow, \mathbf{i}}^{\dagger}, b_{\downarrow, \mathbf{i}}^{\dagger}\right]$, where $a_{\uparrow(\downarrow), \mathbf{i}}^{\dagger}$ and $b_{\uparrow(\downarrow), \mathbf{i}}^{\dagger}$ are the creation operators for sublattice $A$ and $B$ for spin up (down) at site i. $\varepsilon_{\mathbf{i}}=\operatorname{diag}\left(\epsilon_{\mathbf{i}} \mathbf{S}_{\mathbf{0}}, \epsilon_{\mathbf{i}+\mathbf{a}_{1}} \mathbf{s}_{\mathbf{0}}\right)$ is the random potential induced by disorder. Similar to the Hamiltonian of the second model, $t_{c}$ is the nearest-neighbor coupling and $t_{o}$ describes the staggered sublattice potential. In Eq. (5), $t_{e}$ is the exchange energy, which can be achieved by either a magnetic insulator substrate or adsorbing transition-metal atoms (e.g., iron, copper) on graphene. ${ }^{11}$ Finally, $t_{r}$ is the strength of Rashba spin-orbit coupling that has been shown to be fairly strong in graphene. ${ }^{17}$

\section{B. Differential current density and conductance}

In the following, we consider two geometries: ribbon and cylinder. Figures 1(a) and 1(b), respectively, depict the ribbon geometry on the square lattice and honeycomb lattice. In these systems, the finite scattering region [green (or gray in print) region], in which random disorder is considered, is connected to the external reservoir through semi-infinite leads. In the following, we will derive the conductance and the current density in the scattering region.

For the general Hamiltonian $H=\sum_{\sigma \mathbf{i}, \sigma^{\prime} \mathbf{j}} c_{\sigma \mathbf{i}}^{\dagger} \mathbf{H}_{\sigma \mathbf{i}, \sigma^{\prime} \mathbf{j}} c_{\sigma^{\prime} \mathbf{j}}$, local current flowing from site $\mathbf{i}$ with real spin or pseudospin $\sigma$ can be expressed as ${ }^{18}$

$$
\begin{aligned}
\mathbf{J}_{\sigma, \mathbf{i}}(t) & =-e\left\langle\dot{N}_{\sigma, \mathbf{i}(t)}\right\rangle=\frac{i e}{\hbar}\left\langle\left[c_{\sigma, \mathbf{i}}^{\dagger}(t) c_{\sigma, \mathbf{i}}(t), \mathbf{H}\right]\right\rangle \\
& =\frac{e}{\hbar} \sum_{\sigma^{\prime} \mathbf{j}}\left[\mathbf{G}_{\sigma \mathbf{i}, \sigma^{\prime} \mathbf{j}}^{<}(t, t) \mathbf{H}_{\sigma^{\prime} \mathbf{j}, \sigma \mathbf{i}}-\mathbf{H}_{\sigma \mathbf{i}, \sigma^{\prime} \mathbf{j}} \mathbf{G}_{\sigma^{\prime} \mathbf{j}, \sigma \mathbf{i}}^{<}(t, t)\right] \\
& =\sum_{\sigma^{\prime} \mathbf{j}} \mathbf{J}_{\sigma \mathbf{i}, \sigma^{\prime} \mathbf{j}},
\end{aligned}
$$

where $e$ is the electron charge and $\mathbf{G}_{s \mathbf{i}, s^{\prime} \mathbf{j}}^{<}=i\left\langle c_{s^{\prime} \mathbf{j}}^{\dagger} c_{s, \mathbf{i}}\right\rangle$ is the matrix element of the lesser Green's function of the scattering region. Here, $\mathbf{J}_{\sigma \mathbf{i}, \sigma^{\prime} \mathbf{j}}$ is the current from site $\mathbf{i}$ to $\mathbf{j}$. Under dc bias, the current is time independent. After taking Fourier transform, the current $\mathbf{J}_{\sigma \mathbf{i}, \sigma^{\prime} \mathbf{j}}$ can be written as

$$
\mathbf{J}_{\sigma \mathbf{i}, \sigma^{\prime} \mathbf{j}}=\frac{2 e}{\hbar} \int \frac{d E}{2 \pi} \operatorname{Re}\left[\mathbf{G}_{\sigma \mathbf{i}, \sigma^{\prime} \mathbf{j}}^{<}(E) \mathbf{H}_{\sigma^{\prime} \mathbf{j}, \sigma \mathbf{i}}\right] .
$$

Due to the current conservation, $\mathbf{J}_{\sigma, \mathbf{i}}=0$ at each site inside the scattering region. From now on, we will calculate the current density from $\mathbf{J}_{\sigma \mathbf{i}, \sigma^{\prime} \mathbf{j}}$. For the square lattice, it is easy to calculate the current density by summing over all projections of $\mathbf{J}_{\sigma \mathbf{i}, \sigma^{\prime} \mathbf{j}}$ along the $x$ and $y$ directions as was done in Refs. 9, 19, and 20. However, it is much more complicated for graphene because, in the tight-binding representation on graphene, the current can flow from site $\mathbf{i}$ to its nearest- and next-nearestneighbor sites $\mathbf{j}$. Hence, we will use another simple definition ${ }^{21}$ of current density $\mathbf{j}=\rho \mathbf{v}$, where $\rho$ is the charge density and $\mathbf{v}$ is the velocity given by $\mathbf{v}=-i / \hbar(\mathbf{r H}-\mathbf{H r})$. The current density is

$$
\mathbf{J}_{x / y}(\mathbf{i})=-\frac{e}{\hbar} \int \frac{d E}{2 \pi} \operatorname{Re} \sum_{\sigma}\left[\mathbf{G}^{<}\left(\mathbf{r}_{x / y} \mathbf{H}-\mathbf{H} \mathbf{r}_{x / y}\right)\right]_{\sigma \mathbf{i}, \sigma \mathbf{i}},
$$

where $\mathbf{r}$ is a diagonal matrix in the discrete real space. It should be noted that Eq. (8) is valid when site $\mathbf{i}$ is not on the interface between the lead and the scattering region. For these boundary sites, the current density perpendicular to the interface obtained from Eq. (8) should be multiplied by two. This is due to the following reason. When we have a finite scattering, the current is conserved if the self-energy is taken into account. However, for these boundary sites, the current from the lead is not accounted for by Eq. (8).

From the Keldysh equation, the lesser Green's function is related to the retarded and advanced Green's functions

$$
\mathbf{G}^{<}(E)=\mathbf{G}^{r}(E)\left[\sum_{\alpha} \Sigma_{\alpha}^{<}(E)\right] \mathbf{G}^{a}(E),
$$

where the sum index $\alpha=L, R$ denote the left and right semiinfinite leads and $\boldsymbol{\Sigma}_{\alpha}^{<}(E)=i \boldsymbol{\Gamma}_{\alpha}(E) f_{\alpha}(E)$ in Eq. (9) is the lesser self-energy of the lead $\alpha$ with $f_{\alpha}(E)=f_{0}\left(E+e V_{\alpha}\right)$ the Fermi distribution function. Here, $\Gamma_{\alpha}(E)=i\left(\Sigma_{\alpha}^{r}-\Sigma_{\alpha}^{a}\right)$ and $\Sigma_{\alpha}^{a / r}$ is related to the surface Green's function, which can be calculated using a transfer-matrix method. ${ }^{22} V_{\alpha}$ is the external 
bias in the terminal $\alpha$. In general, $G^{<}(E)$ can be divided into equilibrium and nonequilibrium parts ${ }^{19}$

$$
\begin{aligned}
\mathbf{G}^{<}(E)= & \mathbf{G}^{r}(E)\left[i f_{0}(E) \sum_{\alpha} \boldsymbol{\Gamma}_{\alpha}(E)\right] \mathbf{G}^{a}(E) \\
& +\mathbf{G}^{r}(E)\left[i \sum_{\alpha}\left\{f_{\alpha}(E)-f_{0}(E)\right\} \boldsymbol{\Gamma}_{\alpha}(E)\right] \mathbf{G}^{a}(E),
\end{aligned}
$$

where the equilibrium term can only generate persistent current $^{23}$ and does not contribute to the transport, ${ }^{24}$ so it can be dropped out from now on. It is the nonequilibrium term that gives the response to the electron injection from the source lead. Setting source bias $V_{s}=V$ and drain bias $V_{d}=0$, we have

$$
\mathbf{G}^{<}(E)=i \mathbf{G}^{r}(E)\left[f_{s}(E)-f_{0}(E)\right] \boldsymbol{\Gamma}_{s} \mathbf{G}^{a}(E),
$$

where $\boldsymbol{\Gamma}_{s}$ is the linewidth function of source lead. Substituting Eq. (11) into (8), the voltage-dependent differential local current density vector $d \mathbf{J}_{x / y} / d V$ at site $\mathbf{i}$ can be expressed in the form ${ }^{25}$

$$
d \mathbf{J}_{x / y}(\mathbf{i}) / d V=e^{2} \sum_{\sigma} \operatorname{Re}\left[\rho \mathbf{v}_{c, x / y}\right]_{\sigma \mathbf{i}, \sigma \mathbf{i}}
$$

with

$$
\begin{gathered}
\rho=\frac{1}{2 \pi} \mathbf{G}^{r}\left(E_{F}+\mathrm{eV}\right) \boldsymbol{\Gamma}_{s}\left(E_{F}+\mathrm{eV}\right) \mathbf{G}^{a}\left(E_{F}+\mathrm{eV}\right), \\
\mathbf{v}_{c, x / y}=-\frac{i}{\hbar}\left(\mathbf{r}_{x / y} \mathbf{H}-\mathbf{H r}_{x / y}\right),
\end{gathered}
$$

where $\rho$ and $\mathbf{v}_{c, x / y}$ are density matrix with incident energy $E_{F}+\mathrm{eV}$ and velocity matrix in the central scattering region, respectively, and we have assumed the temperature is zero.

When the electron is in the eigenmodes of the semi-infinite lead, there is no scattering, and the linewidth function $\boldsymbol{\Gamma}_{s}$ of the source lead is related to the incident velocity in the form $\hbar \mathbf{v}_{\mathbf{s}}=$ $\mathbf{U}^{\dagger} \Gamma_{\mathbf{s}} \mathbf{U}=\tilde{\boldsymbol{\Gamma}}_{\mathbf{s}}$, ${ }^{26}$ where $\mathbf{v}_{s}$ is the diagonal matrix composed by the nonzero velocity in propagating mode and zero velocity in evanescent mode, and $\mathbf{U}$ is ranked by eigenmodes including the propagating and evanescent modes. Here, $\mathbf{U}$ can be considered as a unitary transformation matrix, which transforms the general Hilbert space into the eigenchannel space of the lead. Then, $\tilde{\boldsymbol{\Gamma}}_{s}$ can be regarded as the incoming velocity matrix in the source lead with relation $\mathbf{v}_{s}=(1 / \hbar) \tilde{\boldsymbol{\Gamma}}_{s}$ (for detail, please refer to Ref. 26). In the calculation, we can pick up only the propagating mode to construct the effective linewidth function $\tilde{\boldsymbol{\Gamma}}_{s}$ as

$$
\overline{\boldsymbol{\Gamma}}_{s}=\sum_{n} \boldsymbol{\Lambda}_{n} \mathbf{v}_{s, n} \boldsymbol{\Lambda}_{n}^{\dagger}
$$

where the sum is taken over propagating modes. $\boldsymbol{\Lambda}_{n}$ is the $n$th column of matrix $\left[\mathbf{U}^{\dagger}\right]^{-1}$, which is related to the $n$th propagating mode. Then, from Eqs. (12) and (13), we can write the differential local current density vector in the $n$th propagating eigenchannel

$$
d \mathbf{J}_{n, x / y}(\mathbf{i}) / d V=\frac{e^{2}}{2} \sum_{\sigma}\left[\operatorname{Re}\left(\rho_{n} \mathbf{v}_{c, x / y}\right)\right]_{\sigma \mathbf{i}, \sigma \mathbf{i}},
$$

where

$$
\rho_{n}=\frac{1}{2 \pi} \mathbf{G}^{r} \boldsymbol{\Lambda}_{s, n} \mathbf{v}_{s, n} \mathbf{\Lambda}_{s, n}^{\dagger} \mathbf{G}^{a} .
$$

As concerns the scalar current flowing into the drain lead $J_{d}$ and the conductance $G=d J_{d} / d V$, we can replace $\mathbf{v}_{c, x / y}$ with $\mathbf{v}_{d}=(1 / \hbar) \tilde{\boldsymbol{\Gamma}}_{d}$ in Eq. (12), where $\boldsymbol{\Gamma}_{d}$ is the effective outgoing velocity matrix of the drain lead similar to the effective incident velocity matrix $\mathbf{v}_{s}=(1 / \hbar) \tilde{\boldsymbol{\Gamma}}_{s}$ in the source lead. Then, considering the representation transformation, from Eqs. (12) and (13), we can get the Landauer-Buttikker formula, ${ }^{27}$ which leads to conductance in zero temperature:

$$
G\left(E_{F}+\mathrm{eV}\right)=\frac{e^{2}}{\hbar} \operatorname{Tr}\left[\operatorname{Re}\left(U \rho U^{\dagger} \tilde{\boldsymbol{\Gamma}}_{d}\right)\right]=\frac{e^{2}}{h} T,
$$

where $T=\operatorname{Tr}\left[\operatorname{Re}\left(\mathbf{G}^{r} \boldsymbol{\Gamma}_{s} \mathbf{G}^{a} \boldsymbol{\Gamma}_{d}\right)\right]$ is the transmission coefficient from the source lead to the drain lead.

\section{NUMERICAL RESULTS AND DISCUSSION}

In the numerical calculations, the energy is measured in the unit of the nearest-neighbor coupling constant $t$. For the first model, $t=B / a^{2}$ with the square lattice constant $a=5 \mathrm{~nm}$. For the second and third models in the honeycomb lattice, $t=2 \hbar v_{F} /(3 a)$ with the carbon-carbon distance $a=0.142 \mathrm{~nm}$ and the Fermi velocity $v_{F}=0.89 \times 10^{6} \mathrm{~ms}^{-1}$ as in a real graphene sample. ${ }^{28}$ The size of the scattering region [the green (or gray in print) region] $M \times N$ is described by integers $M$ and $N$ corresponding to the width and length, respectively. For example, in Fig. 1, the width $W=M a$ with $M=4$, the length $L=N a$ with $M=7$ in Fig. 1(a), and the width $W=$ $M \times 3 a$ with $M=3$, the length $L=N \times \sqrt{3} b$ with $M=7$ in Fig. 1(b).

In all three models Eqs. (2), (4), and (5), the edge state exists in the absence of external magnetic field, which exhibits the quantum anomalous Hall effect. In Fig. 2, we plot the band structure (for calculation, see Ref. 29) and indicate the Fermi energy (the gray lines) in three models. We can see the unidirectional edge states along each edge, i.e., the lower edge [the state in the blue (or dark gray in print) dashed lines] or the upper edge [the state in the red (or light gray in print) lines] in these models. For the first model [Fig. 2(a)], since $e-h$ symmetry is kept, we can focus only on electrons, i.e., only the positive energy is considered. This is different from the $\mathrm{HgTe} / \mathrm{CdTe}$ quantum well, in which $e-h$ symmetry is broken and the edge state is more localized along the edge for the positive energy than the negative energy. In the calculation, we set Fermi energy $E_{F}=0.7 t, A / 2 a=1.35 t, C=3.65 t$, $B / a^{2}=t=27.5 \mathrm{meV}$, and $a=5 \mathrm{~nm}$. For the second model [Fig. 2(b)], the following parameters are used: Fermi energy $E_{F}=0.15 t$, the nearest-neighbor coupling constant $t=$ $27.5 \mathrm{eV}$ that is used as the energy unit in the graphene models, the next-nearest-neighbor coupling strength $t_{n}=0.1 t e^{i \pi / 3}$, and the staggered sublattice potential $t_{o}=0.2 t$. In this model, $e-h$ symmetry is broken by the next-nearest-neighbor coupling $t_{n}$, and the edge states are favored in the hole system (negative energy). In the third model, different from the second one, the next-nearest-neighbor coupling is absent, the Rashba SOC $t_{r}=0.18 t$ and exchange energy $t_{e}=0.2 t$ are considered, and Fermi energy $E_{F}=0.1 t$. Due to the Rashba SOC, the spin 


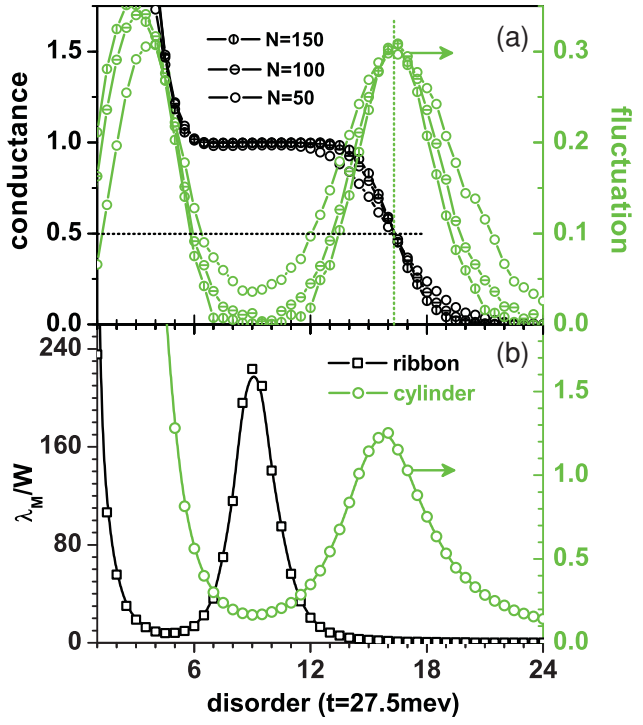

FIG. 3. (Color online) Conductance, fluctuation, and localization length vs disorder for the first model. (a) Conductance (black lines) and fluctuation (green or gray in print lines) in a square lattice with different widths $W=M \times a$. (b) Renormalized localization length of a 2D strip sample (black line) and cylinder sample (green or gray in print line). The width of the strip or the diameter of the cylinder are all $50 a$.

degeneracy is lifted and there are two unidirectional edge states [red (solid) or blue (dashed) lines in Fig. 2(c)] corresponding to different spins. In the second and third models, we find that the staggered sublattice potential $t_{o}$ is important to study the nature of TAI phenomena. First, $t_{o}$ breaks the inversion symmetry, the left [red (solid) line in Fig. 2(b), blue (dashed) line in Fig. 2(c)] and right flowing edge states as well as the bulk states are asymmetrically distributed as shown in Figs. 2(b) and 2(c). As a result, the left- and right-injected current density are distributed differently, although they contribute to the same total current. Second, $t_{o}$ makes the bulk gap smaller, which leads to the coexistence of the robust edge state and bulk states in certain energy window $\Delta E$. For instance, $\Delta E=[0.1,0.5]$ in Fig. 2(b), where only one edge state near the upper edge coexists with the bulk states. In Fig. 2(c), the bulk gap is closed completely (as will be discussed later), which, for this case, makes it harder to induce the TAI phenomena. However this model can give us some insight about TAI phenomena.

In Figs. 3(a) and 4(a), we plot conductance and its fluctuation versus disorder for the first model in a square lattice and the second model in a honeycomb lattice, respectively. In Figs. 3(a), 4(a), and 5, all data are obtained by averaging over 5000 configurations. In Figs. 3(b) and 4(b), we also plot the renormalized localization length $\xi_{M} / W$ of a 2D strip with the width $W=M a$ for the square lattice [Fig. 3(b)] and $W=M \times 3 a$ for the honeycomb lattice [Fig. 4(b)]. Furthermore, in order to highlight the topological nature, we also plot the renormalized localization length $\xi_{M} / W$ in a 2D cylinder sample (where the edge state is removed) with diameter $W$. Here, the Fermi energy $E_{F}$ is set in the bulk state [see Fig. 2]. In the clean system with $w=0$, the conductance is contributed by bulk states and one edge state. When the disorder is introduced, the following observations are in order:

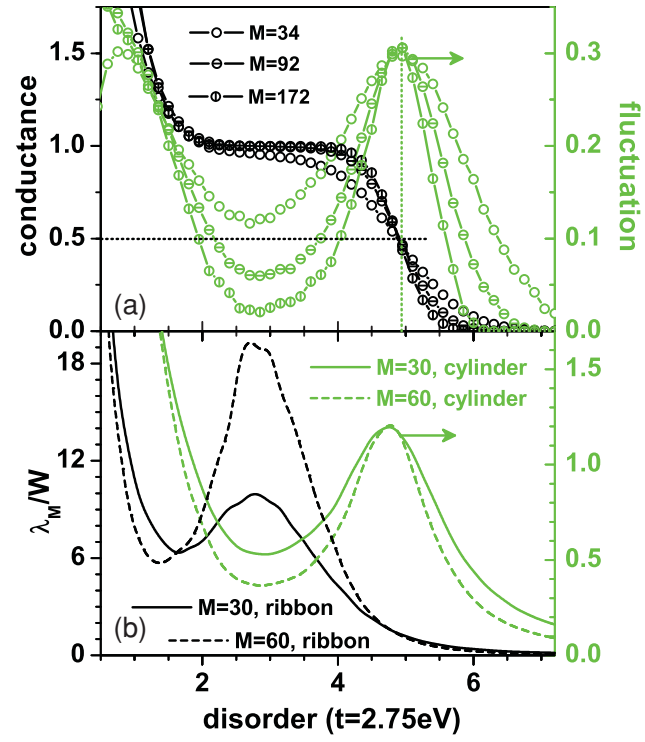

FIG. 4. (Color online) The same as Fig. 3 except that it is for the second model.

(1) The small disorder rapidly suppresses the conductance and enhances its fluctuation. This means that the system is leading to the diffusive regime, in which the bulk extended state is scattered by impurities and gradually becomes localized by disorder. As a result, the localization length decreases accordingly for both ribbon and cylinder samples. (2) At moderate disorders, in the ribbon sample, the conductance stops decreasing and develops into a quantized plateau and a new phase occurs. At the same time, the fluctuation is reduced to almost zero, indicating the formation of either a bulk insulator or metallic phase. The abruptly increased localization length (black lines) in Figs. 3(b) and 4(b) clearly indicates the metallic phase induced by edge state. For the cylinder sample, however, because of the absence of the edge state, the system can only develop into the bulk insulator, so the localization length continues to decrease. Our results show that the wider the ribbon is, the larger $\xi_{M} / W$ in ribbon geometry and the smaller $\xi_{M} / W$ in cylinder geometry [see Fig. 4(b)]. Therefore, concerning $\xi_{M} / W$, the peak structure in ribbon geometry and the valley structure in cylinder geometry clearly indicate the topological nature of the TAI phenomena. (3) When the disorder is strong, the quantized conductance plateau in the ribbon sample starts to deteriorate and its fluctuation begins to increase. This shows that the edge state will be destroyed at the strong disorder and the system will enter the insulating regime. For the cylinder sample, the system transforms from band insulator to Anderson insulator. The peak of $\xi_{M} / W$ indicates the transition points. (4) At the critical disorder strength $w_{c}$, MIT occurs. $w_{c}$ is marked in Figs. 3 and 4 by the vertical green (gray in print) dotted lines, where conductance $G=0.5 \frac{e^{2}}{h}$ and $\xi_{M} / W$ is between 1 to 1.5 . For the cylinder sample, $\xi_{M \rightarrow \infty}$ diverges at $w_{c}$. It should be noted that despite the different topological nature, the MIT occurs at nearly the same $w_{c}$ for ribbon and cylinder samples. (5) In the quantized conductance regime, by increasing the sample size, the quantized plateau is much wider with smaller fluctuation. This is not surprising since, for the larger sample, the bulk state is scattered more 


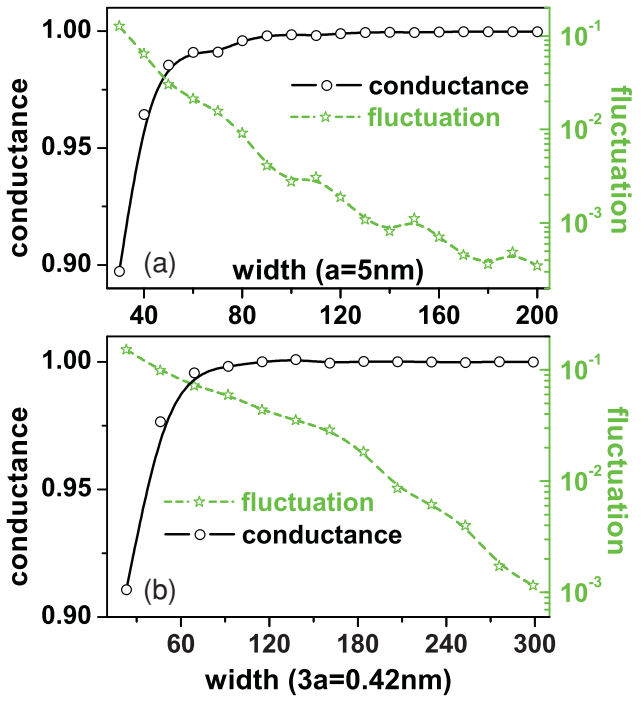

FIG. 5. (Color online) Conductance (black lines) and fluctuation (green or gray in print lines) of a 2D square sample vs sample size in the first model for the fixed disorder strength $w=9$ (a) and in the second model for the fixed disorder strength $w=2.25$ (b).

frequently while the overlap of the opposite unidirectional edge states is smaller. This size effect is clearly seen in Fig. 5, in which the quantized conductance and its fluctuation in the midpoint $[w=9$ for Fig. 5(a) and $w=2.25$ for Fig. 5(b)] of conductance plateaus are plotted versus the width of the square sample for the first and second models. We can see that, with the increasing of the sample size, the conductance eventually saturates to the quantized value; its fluctuation, however, continues to drop. It is expected that, when the size is large enough, the complete quantized plateau will be formed with no conductance fluctuation.

Up to now, we have confirmed that, in addition to the $\mathrm{HgTe} / \mathrm{CdTe}$ quantum well, the disorder-induced quantized conductance (TAI) can also occur in other systems such as the Dirac model and the graphene system. Hence, it is interesting to examine the common features of TAI phenomena in different systems. For this reason, we study the scattering process in different systems through monitoring the local current density.

First, we study the Dirac model. Due to the inversion symmetry in the Dirac model, the left- and right-injected currents are equivalent, hence, we focus only on the leftinjected current. We have set the Fermi energy to be $E_{F}=0.7$ for Fig. 6. In Figs. 6 and 8, all data are obtained by averaging over 1000 configurations. For $E_{F}=0.7$, there are several transmission channels and we have studied the first three transmission channels: one is edge state and the other two are bulk states. In Figs. 6(a)-6(c), we plot the left-injected local differential current density distribution from the lowest three transmission channels, including the edge channel [Fig. 6(c)] and the first [Fig. 6(b)] and second [Fig. 6(a)] bulk channels in a square sample (between two vertical gray lines). For the clean system with $w=0$ (the left column), the system is ballistic and there is no scattering. So, the current density from all transmission channels is distributed along the transport direction. For the left column of Fig. 6, we can see the right-going edge state on the upper edge and the extended bulk

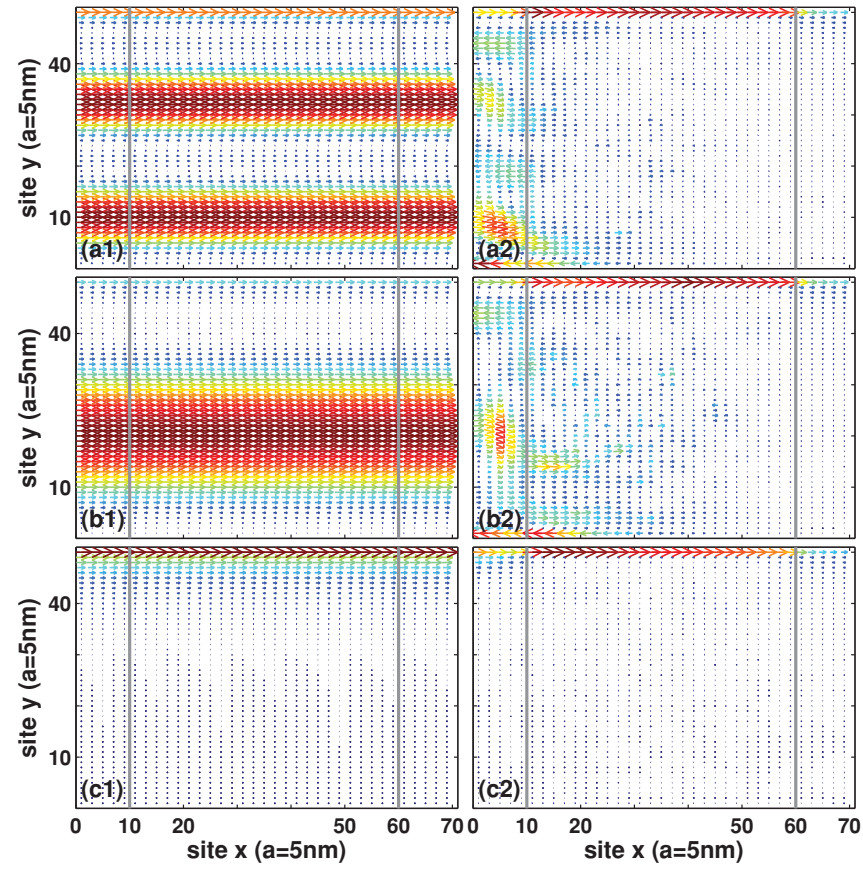

FIG. 6. (Color online) Left-injected local differential current density vector distribution for the lowest three subchannels in the first model. The direction and the length of the arrow in every site show the direction and the magnitude of the current density vector in this site. Concerning a visual effect, the magnitude of the current vector is also expressed by different color. With the increasing magnitude, the color changes from blue (light) to red (dark). The left and right columns are corresponding to the clear sample and dirty sample with disorder strength $w=6$.

state with one or two transverse peaks in the current density. Note that the incoming eigenchannels are classified according to the lead. When disorder is present, due to the mode mixing, the electron in the edge channel can be scattered into bulk channels and vice versa. As a result, the eigenchannels of the lead are no longer the eigenchannels of the whole system. Corresponding to the left column of Fig. 6, in the right column, we plot the current density distribution at the entrance of the TAI (quantized conductance) with $w=6$. We can see that the right-going edge current in all transmission channels of the lead are well protected.

In order to study the nature of the edge state in the presence of disorders, we have calculated the eigenspectrum of the transmission matrix $\Gamma_{L} G^{r} \Gamma_{R} G^{a} \cdot{ }^{30}$ In Fig. 7(b), we plot all the transmissions of eigenchannels for the the first and second models. For comparison, in Fig. 7(a), we also plot the eigentransmissions of the $\mathrm{HgTe} / \mathrm{CdTe}$ quantum well in which (except for the terms in the first model) the term of $D \mathbf{k} \cdot \mathbf{k}$ is also included. Here, we set $D / a^{2}=0.75 t$ with $t=B / a^{2}$ and the other parameters are the same as in the first model. In Fig. 7, all data are obtained by averaging over 100 configurations. We found that there is always one eigenvalue that is nearly equal to one that is identified as the edge state by examining its current density profile. It shows that, due to the topological nature, the edge state is always protected during the transport, although it may be scattering into the bulk channels of lead. From Fig. 7, we also found that the eigentransmissions of the first and 


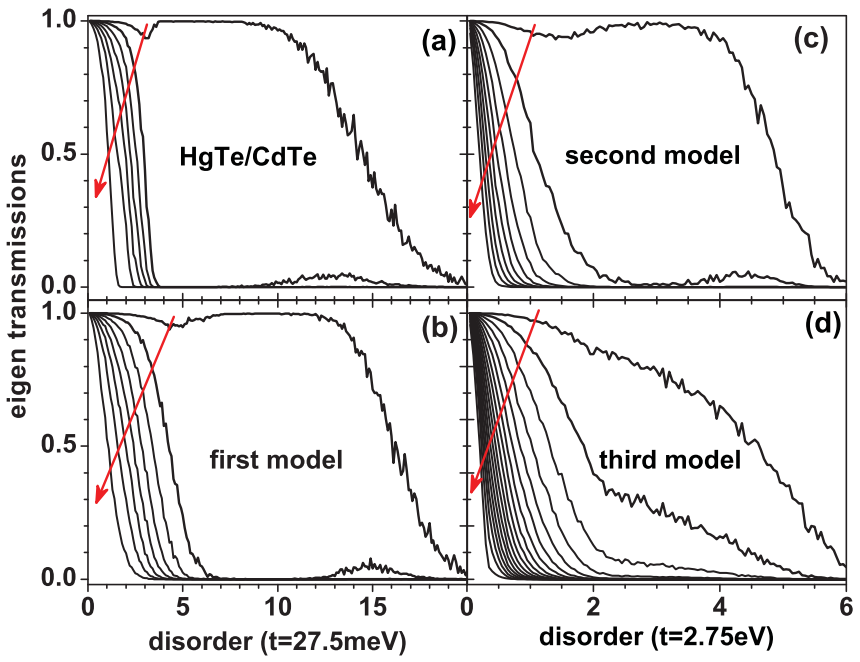

FIG. 7. (Color online) Transmissions of system eigenchannels in a square sample for the first model [(b), the width $W=100 a$ ], the second model [(c), the width $W=92 \times 3 a$ ], the third model [(d), the width $W=100 \times 3 a$ ], and the $\mathrm{HgTe} / \mathrm{CdTe}$ quantum well [(a), the width $W=100 a$ ]. Different lines along the (red) arrow correspond to the eigen-edge channel, the first to $n$th eigen-bulk channels.

second models in this paper exhibit the same properties as those in the $\mathrm{HgTe} / \mathrm{CdTe}$ quantum well. It strongly suggests that they share the common feature.

Next, we examine the graphene model. We set $E_{F}=0.15$ for Fig. 8. In the graphene model, the staggered sublattice potential is considered, which breaks the inversion symmetry, and the configurations of left- and right-injected current density are different. In a clean system, the left- (right-) injected current is contributed by the states that have a positive (negative) group velocity, i.e., $\partial E / \partial k_{x}>0(<0)$. For the fixed Fermi energy, the crossing of Fermi energy and energy band determines the momentum $k$ of all propagating states. From the band structure of the graphene model [Figs. 2(b) and 2(c)], we can see that the edge state is absent for the right-propagating

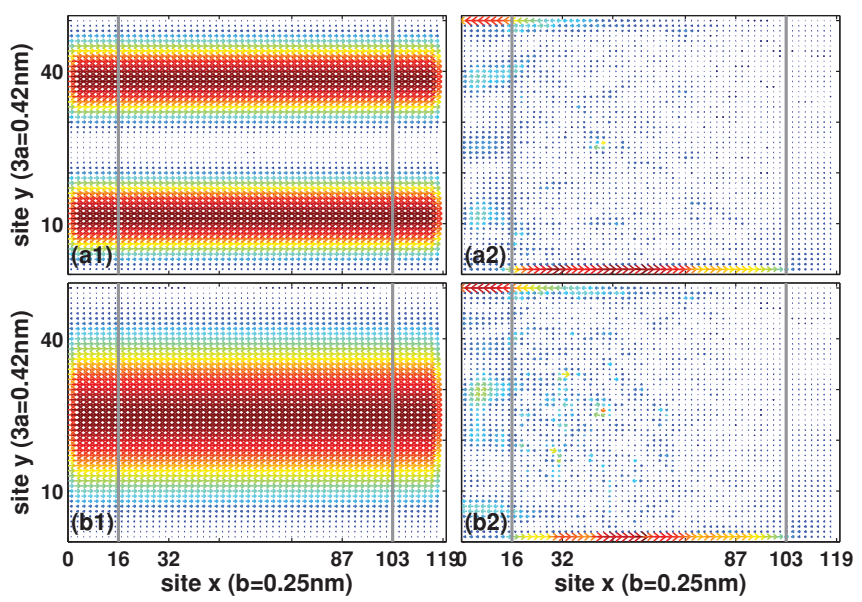

FIG. 8. (Color online) (a), (b): Left-injected local differential current density vector distribution for the lowest two subchannels in the second model. The left and right columns are corresponding to the clear sample and dirty sample with disorder strength $w=1.8$. band in the second model, while in the third model, it is absent for the left-propagating state.

In Fig. 8, we plot the left-injected current density in the second model. We can see that, for the left-injected current, although edge current is absent in clean limit, it emerges when disorder is turned on. This can be understood as follows. ${ }^{10}$ When the disorder is present, the effective medium theory similar to the one in Ref. 10 shows that the parameters in the second model such as $t_{n}$ and $E_{F}$ are all renormalized and depend on disorder strength. After renormalization due to the disorder, the new Fermi level (renormalized) is inside the bulk gap of the new band structure giving rise to a true edge state.

We have also calculated the right-injected current density. We found that the disorder also eliminates the difference between states with different momentum $k$ due to its random distribution. Hence, although the profiles of left- and rightinjected current densities are different in the clean system, when the moderate disorder is considered, they tend to be the same since the bulk states are killed and only the edge state survives [see Figs. 7 and 8]. Of course, the left-going and right-going edge states are along the opposite edge. Similar to the first model, in the clean limit, the current density in all eigenchannels is present and uniformly distributed along the transport direction, except they have different profiles for leftand right-injected current density.

From Figs. 6 and 8, it seems that the phenomena of disorder-induced quantized conductance can occur in any system as long as the quantum anomalous Hall effect is present. However, it should be noted that, in the first and second models, the edge state is robust, which gives rise to the quantized conductance plateau. In the following, we will study another quantum anomalous Hall system, which is the third model [Eq. (5)]. Similar to the second model, the configuration of the left- and right-injected current density are different in the clean limit due to the inversion asymmetry, hence, we focus only on the left-injected case in which the edge current is present in the clean system. In the third model, the bulk energy gap is closed by the staggered sublattice potential, and the edge state may coexist with bulk states but can not exist for any incident energy. For this reason, the edge state is not as robust as in the first and second models. Furthermore, due to inversion asymmetry, one of the two unidirectional edge states turns into the bulk state [see Fig. 2(c)]. So, the disorder-induced conductance must be $e^{2} / h$ if there is any.

Because the edge state is not as robust, the backscattering edge current is strong. Although there is one unambiguous unidirectional edge state, it is drastically reflected by the interface scattering. Hence, the edge current can not penetrate deeply into the scattering region when moderate disorder strength is presented. On the other hand, the edge state that enters into the scattering region can always survive in a moderate disorder. As a result, the conductance can not be quantized but rather flattened by the moderate disorder, which is different from the first and second models, but they share the same feature, which can also be seen from Figs. 7(c) and $7(\mathrm{~d})$.

In Figs. 7(c) and 7(d), we plot the system eigentransmission coefficients of the second and third models. We can see that, for all the models (the first, second, and third models and the $\mathrm{HgTe} / \mathrm{CdTe}$ quantum well), the transmission coefficients 
of the eigen-bulk state are rapidly reduced to zero at weak disorders, while the eigen-edge state can sustain moderate disorders. Figures 7(a)-7(c) are examples of robust eigen-edge state that are intact at large disorders. For Fig. 7(d), the eigen-edge state is not as robust but the bulk states disappear much faster. Given the fact that the first, second, and third models are similar to the $\mathrm{HgTe} / \mathrm{CdTe}$ quantum well, we can not rule out the possibility that, for a suitable set of parameters, the edge state survives when the bulk states are killed by disorders. From the above discussion, we can conclude that, in the system where the robust edge state and bulk state coexist, if the edge state is robust enough against the disorder while the bulk states are killed at certain disorders, quantized conductance can be formed and the TAI phenomena appears.

\section{CONCLUSION}

In summary, the disorder effect was studied in several quantum anomalous Hall systems with different symmetries, i.e, $e$ - $h$ symmetry and time-reversal symmetry. We found that, in addition to the $\mathrm{HgTe} / \mathrm{CdTe}$ quantum well, the modified Dirac model and the graphene system with next-nearest coupling and staggered sublattice potential also exhibit TAI. For the graphene sheet with Rashba spin-orbit interaction as well as an exchange field, our results show some signature of TAI, but are not conclusive. Our numerical results show that for the Fermi energy outside of the bulk energy gap, the edge state and bulk state often coexist in clean systems or weak disordered systems. In a moderate disorder, the interband scattering happens. If the edge state is robust enough to resist the interface scattering, it can penetrate deep into the scattering region where the edge state and bulk state coexist for all of the incoming eigenchannels due to the mode mixing. Due to the topological nature, the edge state can survive when bulk states are completely killed, leading to TAI. Analyzing the eigenspectrum of the whole scattering system, we found that the eigenchannel corresponding to the edge state is well protected, while the other bulk channels are completely killed by disorders. Without the bulk states, the unidirectional edge state located in the up or down edge can not be transformed to the opposite edge, and the backscattered current is then prohibited. As a result, the system exhibits the TAI phenomenon with the quantized conductance plateau formed.

\section{ACKNOWLEDGMENT}

We gratefully acknowledge the financial support by a RGC grant (No. HKU 705409P) from the Government of HKSAR and Excellent young scholars Research Fund of Beijing Institute of Technology. *jianwang@hkusua.hku.hk

${ }^{1}$ E. Abrahams, P. W. Anderson, D. C. Licciardello, and T. V. Ramakrishnan, Phys. Rev. Lett. 42, 673 (1979).

${ }^{2}$ S. Murakami, N. Nagaosa, and S. C. Zhang, Science 301, 1348 (2003); Phys. Rev. B 69, 235206 (2004); J. Sinova, D. Culcer, Q. Niu, N. A. Sinitsyn, T. Jungwirth, and A. H. MacDonald, Phys. Rev. Lett. 92, 126603 (2004); L. Sheng, D. N. Sheng, and C. S. Ting, ibid. 94, 016602 (2005).

${ }^{3}$ Z. Qiao, Y. Xing, and J. Wang, Phys. Rev. B 81, 085114 (2010).

${ }^{4}$ D. N. Sheng and Z. Y. Weng, Phys. Rev. Lett. 80, 580 (1998).

${ }^{5}$ F. D. M. Haldane, Phys. Rev. Lett. 61, 2015 (1988).

${ }^{6}$ M. Onoda and N. Nagaosa, Phys. Rev. Lett. 90, 206601 (2003);

M. Onoda, Y. Avishai, and N. Nagaosa, ibid. 98, 076802 (2007);

H. Obuse, A. Furusaki, S. Ryu, and C. Mudry, Phys. Rev. B 76, 075301 (2007).

${ }^{7}$ J. Li, R. L. Chu, J. K. Jain, and S. Q. Shen, Phys. Rev. Lett. 102, 136806 (2009).

${ }^{8}$ C.-X. Liu, X.-L. Qi, H. J. Zhang, X. Dai, Z. Fang, and S.-C. Zhang, Phys. Rev. B 82, 045122 (2010); S. Raghu, X.-L. Qi, C. Honerkamp, and S.-C. Zhang, Phys. Rev. Lett. 100, 156401 (2008); B. A. Bernevig and S.-C. Zhang, ibid. 96, 106802 (2006); C. L. Kane and E. J. Mele, ibid. 95, 226801 (2006).

${ }^{9}$ H. Jiang, L. Wang, Q.-F. Sun, and X. C. Xie, Phys. Rev. B 80, 165316 (2009).

${ }^{10}$ C. W. Groth, M. Wimmer, A. R. Akhmerov, J. Tworzydło, and C. W. J. Beenakker, Phys. Rev. Lett. 103, 196805 (2009).
${ }^{11}$ Z. Qiao, S. A. Yang, W. Feng, W.-K. Tse, J. Ding, Y. Yao, J. Wang, and Q. Niu, Phys. Rev. B 82, 161414 (2010).

${ }^{12}$ R. Nandkishore and L. Levitov, Phys. Rev. B 82, 115124 (2010); Y.-F. Hsu and G.-Y. Guo, ibid. 82, 165404 (2010).

${ }^{13}$ D. N. Sheng, Z. Y. Weng, L. Sheng, and F. D. M. Haldane, Phys Rev. Lett. 97, 036808 (2006); D. Xiao, M. C. Chang, and Q. Niu, Rev. Mod. Phys. 80, 1355 (2008).

${ }^{14}$ B. A. Bernevig, T. L. Hughes, and S.-C. Zhang, Science 314, 1757 (2006); C.-X. Liu, X.-L. Qi, X. Dai, Z. Fang, and S.-C. Zhang, Phys. Rev. Lett. 101, 146802 (2008).

${ }^{15}$ Electronic Transport in Mesoscopic Systems, edited by S. Datta (Cambridge University, Cambridge, UK, 1995).

${ }^{16}$ L. Sheng, D. N. Sheng, C. S. Ting, and F. D. M. Haldane, Phys. Rev. Lett. 95, 136602 (2005).

${ }^{17}$ Y. S. Dedkov, M. Fonin, U. Rüdiger, and C. Laubschat, Phys. Rev. Lett. 100, 107602 (2008).

${ }^{18}$ A.-P. Jauho, N. S. Wingreen, and Y. Meir, Phys. Rev. B 50, 5528 (1994).

${ }^{19}$ Y. Xing, J. Wang, and Q.-F. Sun, Phys. Rev. B 81, 165425 (2010).

${ }^{20}$ J. Li and S.-Q. Shen, Phys. Rev. B 76, 153302 (2007).

${ }^{21}$ B. K. NikolićL, P. Zârbo, and S. Souma, Phys. Rev. B 73, 075303 (2006).

${ }^{22}$ D. H. Lee and J. D. Joannopoulos, Phys. Rev. B 23, 4997 (1981); 23, 4988 (1981).

${ }^{23}$ Here, the persistent current is due to the magnetic vector potential $A$. If $A=0$, equilibrium current is zero because the left and right 
leads contribute opposite current density considering the symmetry properties (Ref. 21).

${ }^{24}$ A. Cresti, G. Grosso, and G. P. Parravicini, Phys. Rev. B 69, 233313 (2004).

${ }^{25}$ The expression should be modified when the site is on the boundary of the scattering region.

${ }^{26}$ P. A. Khomyakov, G. Brocks, V. Karpan, M. Zwierzycki, and P. J. Kelly, Phys. Rev. B 72, 035450 (2005).

${ }^{27}$ T. P. Pareek, Phys. Rev. Lett. 92, 076601 (2004);

Y. Xing, Q.-F. Sun, and J. Wang, Phys. Rev. B 73,
205339 (2006); T. P. Pareek, Phys. Rev. Lett. 80, 235411 (2009).

${ }^{28} \mathrm{~K}$. Novoselov et al., Nature (London) 438, 197 (2005); Y. Zhang, Y.-W. Tan, H. L. Stormer, and P. Kim, ibid. 438, 201 (2005); K. Novoselov et al., Nat. Phys. 2, 177 (2006); J. R. Williams, L. Dicarlo, and C. M. Marcus, Science 317, 638 (2008).

${ }^{29}$ Y. Hatsugai, Phys. Rev. B 48, 11851 (1993).

${ }^{30}$ Z. Qiao, J. Wang, Q.-F. Sun, and H. Guo, Phys. Rev. B 79, 205308 (2009). 